\title{
Effects of various treatment regimens on the survival of HER2-positive breast cancer patients with brain metastasis
}

\author{
Shantal Edirappuli \& Emma Beddowes \\ University of Cambridge, UK \\ correspondence to sde29@cam.ac.uk
}

Cite as: Edirappuli S \& Beddowes E. (2020) Effects of various treatment regimens on the survival of HER2-positive breast cancer patients with brain metastasis. The Physician 6(1)c12 DOI: 10.38192/1.6.1.c12

\section{Background:}

Overexpression of HER2 (human epidermal growth factor receptor 2) occurs in around 20\% of breast cancers. This particular subtype is known to be very aggressive and had the worst prognosis prior to the introduction of targeted therapy. The development of anti-HER2 therapies such as trastuzumab and pertuzumab have shown to dramatically improve the prognosis of patients with HER2-positive breast cancer. Despite these improved prognoses with newer drug therapies, the development of brain metastases and subsequent intracranial progression is often fatal in patients. The traditional options for treatment of brain metastases include neurosurgical resection, whole brain radiotherapy and stereotactic radiosurgery. This study aims to provide an analysis of the various treatment regimens that are employed and their effects on overall survival in patients.

Methods:

27 relevant patients (those with HER2-positive breast cancer and brain metastasis) were extracted from the ongoing DETECT study based in Cambridge. The effect of various parameters, including treatment regimens, was analysed using Kaplan-Meier curves.

Results:

The results showed that patients who received neurosurgical intervention had a statistically significant greater overall survival time compared to patients who had not received any neurosurgical intervention (median survival $=40.80$ vs 12.35 months, $p=0.011$ ). The data further showed that neither radiotherapy nor drug therapies (chemotherapy, hormonal therapy or anti-HER2 therapy) significantly affected the overall survival.

Conclusions:

Of the current therapies employed by clinicians, neurosurgical intervention alone correlates with a significantly increased overall survival in patients. The explanations for this are varied but not limited to the specificity of this treatment in targeting intracranial tumours. Additionally, the clinical decision-making process for which patients may be considered eligible for surgery selects for those with a better prognosis. Future options for therapy include prophylactic cranial irradiation which may prove effective in targeting the, often-fatal, intracranial progression of this cancer. 